\title{
The Use of Electroanatomic Mapping Systems to Reduce Fluoroscopic Exposure in Pediatric Ablations
}

\author{
GEORGE M. MCDANIEL, MD, MS \\ Division of Pediatric Cardiology, Department of Pediatrics, University of Virginia, Charlottesville, VA
}

\begin{abstract}
Concerns about the long-term effects of ionizing radiation for both patients and electrophysiology laboratory personnel combined with the development of electroanatomic mapping systems have made it possible to perform ablation of dysrhythmia substrates with minimal or no use of fluoroscopy. This article reviews the current literature demonstrating the safety and efficacy of this technique in pediatric patients. While most reports are retrospective and single-center studies, there is a growing interest in developing multicenter studies to further refine this technique.
\end{abstract}

KEYWORDS. electroanatomic mapping systems, fluoroscopy, pediatric ablations.
ISSN 2156-3977 (print) ISSN 2156-3993 (online)

(C) 2011 Innovations in Cardiac Rhythm Management

\section{Introduction}

Electroanatomic three-dimensional mapping systems were developed to aid in the mapping and ablation of tachycardia substrates by offering a more useful image of cardiac chamber dimension, and catheter position within that chamber, than fluoroscopy alone. However, longstanding concerns over the effects of ionizing radiation exposure on patients and laboratory personnel quickly led groups to explore the capacity of these systems to safely and effectively allow the minimization of exposure to fluoroscopy in adult patients. ${ }^{1-5} \mathrm{~A}$ logical progression of this trend was to attempt the elimination of fluoroscopy altogether, even for more complex ablations. ${ }^{6}$

While minimizing fluoroscopy is prudent for all persons involved in an ablation, the possibility of a procedure without fluoroscopy is particularly appealing in specific populations. ${ }^{1,2}$ One such population is pregnant women where the potential negative effects of the exposure of a fetus to ionizing radiation have the opportunity to develop and be compounded over a lifetime. ${ }^{7}$

The author reports no conflicts of interest for the published content. Manuscript received October 2, 2011, final version accepted October 22, 2011.

Address correspondence to: George M. McDaniel, MD, MS, Division of Pediatric Cardiology, Department of Pediatrics, University of Virginia, 1215 Lee St., Charlottesville, VA 22908. E-mail: GMM4D@hscmail.mcc.virginia.edu
The same argument holds true for the performance of mapping and ablation procedures in the pediatric population, where it may become necessary to perform these procedures in children under 1 year of age. ${ }^{8}$ Although the relative increase in long-term risk of malignancy from ionizing radiation exposure is felt to be small, it remains unknown. ${ }^{9-11}$

Additionally, patients with congenital heart disease often have dysrhythmias. Owing to anatomic alterations and multiple substrates, procedures are often longer than in patients with structurally normal hearts necessitating longer imaging times. Also, as these patients are likely to have multiple procedures, it is of particular importance to minimize radiation use at any one procedure. Whereas there are a number of studies on the use of electroanatomic mapping systems in adult patients, this review will focus on clinical studies that have be performed in pediatric patients with or without congenital heart disease.

\section{Electroanatomic mapping systems}

The development of relatively inexpensive computer systems that have the capacity to process signals rapidly has allowed the development of mapping systems that reliably localize intracardiac catheter positions. One such system is the CARTO system (Biosense Webster, Diamond Bar, CA). 
The system uses a magnet mounted under the catheterization table coupled with a proprietary catheter placed in the intracardiac chamber of interest. The magnet under the table locates the catheter tip in three-dimensional space when an endocardial signal is measured and the system stores the spatial and electrical information. The computer then constructs a virtual three-dimensional electroanatomic map of the chamber (Figure 1). The catheter tip location within the mapped space is displayed on the computer screen allowing catheter manipulation without fluoroscopy. In current versions of CARTO, real-time structural data from intracardiac ultrasound (CartoSound) or changes in impedance (CARTO 3) can be integrated to refine the displayed image.

The LocaLisa (Medtronic Inc, St. Paul, MN) system was used in early studies of non-fluoroscopic procedures. The technology was acquired by Endocardial Solutions, which subsequently was acquired by the St. Jude Medical corporation. The technology was incorporated into the EnSite NavX system (St. Jude Medical, St Paul, MN). Patches are placed on the skin and used to generate an electrical signal sensed by the catheters inside the body. A catheter in a stable position is used as a reference for creating the geometry. The ablation catheter is manipulated within the chamber of interest and when contact with the wall is demonstrated by the measured electrical signal, the catheter position is recorded (Figure 2). Electrical impedance changes are sensed by the system and indicate a change in catheter position, and the system then displays the new catheter position. The system then can simultaneously track 64 separate electrodes on up to 12 different catheters. Their positions are displayed relative to each other within a virtual representation of the cardiac chamber(s) of interest.

Although both the CARTO and EnSite systems produce a virtual image of the cardiac chambers and catheter(s), there are differences in them, some of which are summarized in Table 1. However, with both systems, there are increased equipment costs associated with both the CARTO and NavX systems. Because the CARTO system is based on changes within a magnetic field, the ablation catheter has to be magnetically active. This proprietary catheter is more expensive to purchase than commonly used ablation catheters. The NavX system is compatible with most commonly used catheters, but the patches applied to the skin are one-time use only and must be purchased for every case. It is difficult to make a global statement about the absolute cost increase as there may be differences based on volume and specific center preferences.

\section{Clinical studies}

In 2002, the first publication of the elimination of fluoroscopy for ablation procedures in the pediatric population was by Drago and colleagues. ${ }^{12}$ In their study, they used the CARTO system to successfully ablate accessory pathways located in the right atrium in 21 pediatric patients, mean age 11.3 years, with WolffParkinson-White syndrome. They demonstrated an acute success rate of $95 \%$ without complications, comparable to ablation using standard fluoroscopic

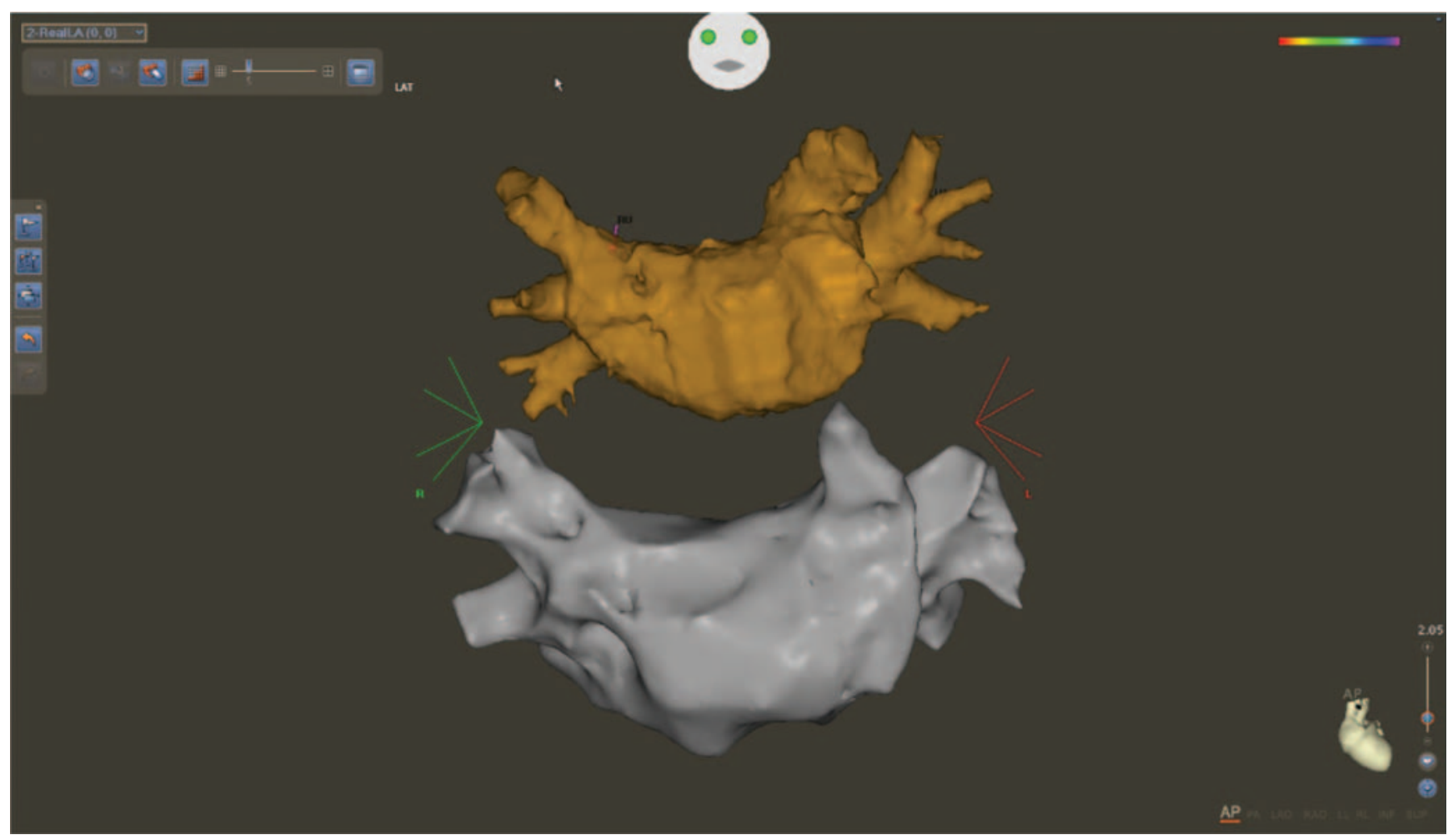

Figure 1: Geometry of a left atrium displayed on a CARTO system. Courtesy of Mr. Randolph Ellis, Biosense Webster, Inc. 


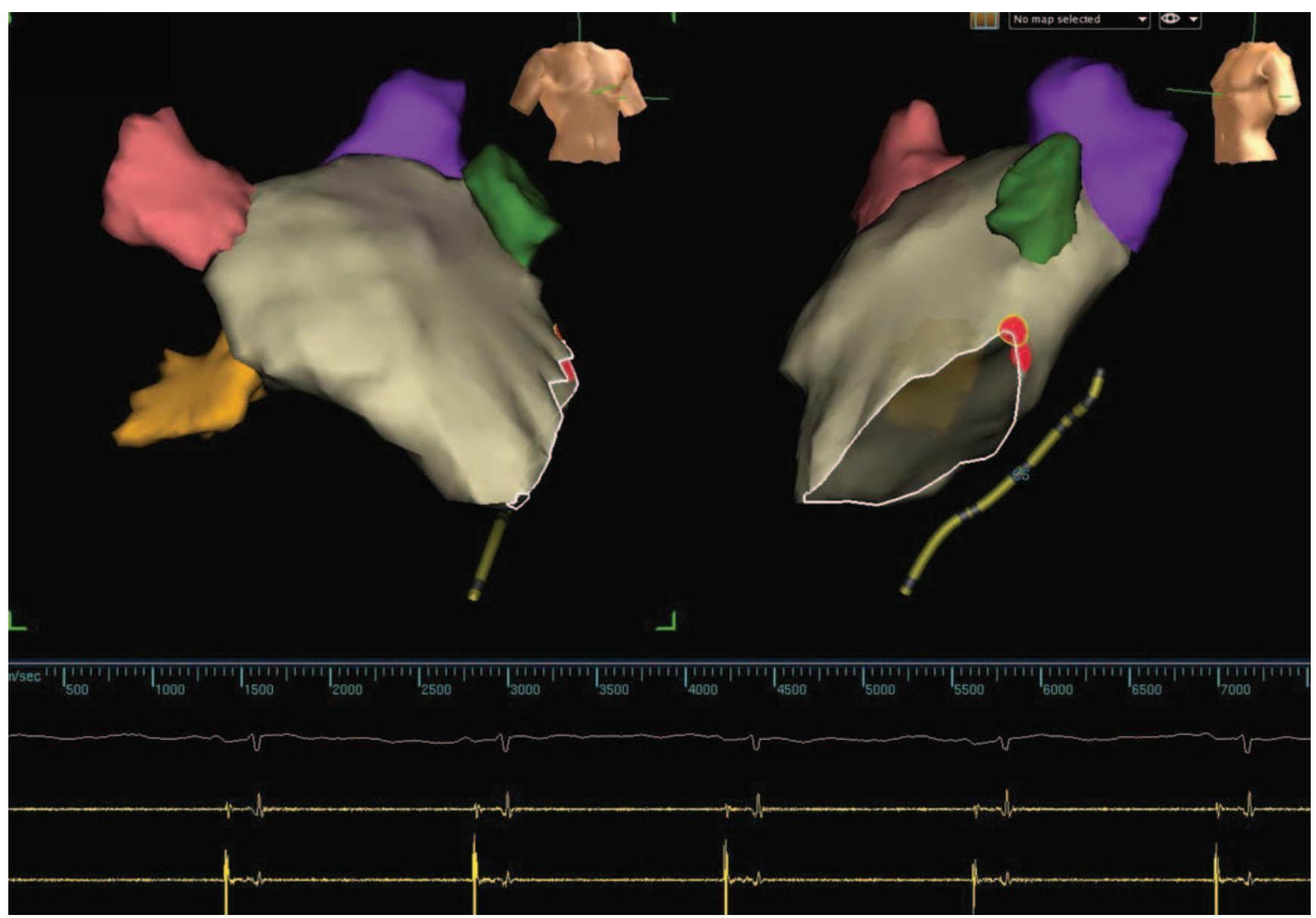

Figure 2: Geometry of a left atrium displayed on an EnSite NavX system. The opening represents the position of the mitral annulus. The position of a decapolar catheter in the coronary sinus is also displayed. The red circles represent the site of ablation of an accessory pathway. Courtesy of Mr. John Hobby, St. Jude Medical S.C., Inc.

techniques. ${ }^{13}$ Further, they noted that fluoroscopy use was decreased in their first 10 patients, and that after the thirteenth patient, no fluoroscopy was required to perform the ablation.

Using the LocaLisa system, further studies on the impact of electroanatomic mapping systems on the reduction of fluoroscopy in pediatric patients were reported. ${ }^{14}$ Patients were divided into two groups, those who had their procedures done with fluoroscopy and those done with the addition of computer-assisted navigation. The group assignment was not random but temporal, in that the group whose procedures were performed with fluoroscopy alone comprised patients treated prior to the acquisition of the computer-assisted navigation system. There were no other demographic differences or tachycardia substrate differences seen in the two groups. Procedural success rates and complication rates were not different between the groups. However, there was a significant reduction in mean fluoroscopic times with the addition of the electroanatomic mapping system $(17.2 \pm 12.6$ versus $37.3 \pm 21.3 \mathrm{~min}, \mathrm{p}<0.0001)$. The authors also reported the use of fewer energy applications in the group with electroanatomic mapping assistance $(9.0 \pm 8.5$ versus $12.3 \pm 12.2, \mathrm{p}<0.02)$. Furthermore, analysis of subgroups stratified by tachycardia mechanism and accessory pathway location demonstrated a significant

Table 1: Comparison of some features of the CARTO and EnSite NavX systems

\begin{tabular}{|c|c|c|}
\hline & CARTO & NavX \\
\hline Simultaneous display of multiple catheters & No (yes in CARTO 3 ) & Yes \\
\hline $\begin{array}{l}\text { Displayed location may be altered by inadvertent impedance changes such as } \\
\text { irregular respirations }\end{array}$ & No & Yes \\
\hline Displayed location may be altered by small, inadvertent movement of the reference & No & Yes \\
\hline Integrates with other imaging modalities (CT, MRI) & Yes & Yes \\
\hline
\end{tabular}

CT: computed tomography; MRI: magnetic resonance imaging. 
decrease in total procedure time in patients whose mechanism was an accessory pathway not located near the atrial septum.

As an investigation of safety and efficacy of a nonfluoroscopic technique, Tuzcu ${ }^{8}$ also published the contemporary experience of ablating right-sided substrates in pediatric patients at his institution. His study group consisted of 24 consecutive patients who underwent electrophysiology study and ablation where the intention was to use no fluoroscopy for the entire procedure. For a comparison group, 19 patients who had undergone right-sided ablations prior to the acquisition of their EnSite system were used. There were no significant differences in age and tachycardia substrates between the two groups. The acute success rate in the nonfluoroscopy group was $92 \%$. The acute success rate in the fluoroscopy group was $84 \%$, but the difference was not statistically different. The recurrence rate was not statistically different between the two groups $(19 \%$ in the non-fluoroscopy group and 13\% in the fluoroscopy group). The number of lesions delivered was not different between the two groups. There were no complications reported in either group. The mean procedural time was not statistically different between the groups, $193.5 \pm$ $80 \mathrm{~min}$ for the non-fluoroscopy group and 208.2 \pm $64.5 \mathrm{~min}$ for the fluoroscopy group. From the report it was clear that the author has extensive experience in the use of the technique for left- or right-sided substrates, but as a brief period of fluoroscopy was used for left-sided substrates these patients were excluded from the report.

In 2007, Smith and Clark ${ }^{15}$ published their experience using the EnSite system in NavX mode to accomplish ablation in children with minimal fluoroscopy use. Their study cohort consisted of 30 patients who had undergone ablation using the EnSite system. Patients who had undergone ablation in that laboratory prior to the acquisition of the EnSite system were used as a control group. They were matched for age and arrhythmia substrate. Mean fluoroscopy time for the study group was $1.05 \mathrm{~min}$ compared with $21.4 \mathrm{~min}$ for the control group $(p<0.05)$. There was no significant difference in procedure time. More interesting, however, was that 24 of the 30 study patients, $80 \%$, required no fluoroscopy. Of the six patients who required fluoroscopy, five had left-sided pathways. That laboratory uses predominantly the transseptal approach to access the mitral annulus and fluoroscopy was used to perform the transseptal puncture.

A similar experience with the EnSite system in pediatric patients was reported by Papagiannis et al. ${ }^{16}$ Their retrospective study consisted of two groups of 40 patients, matched for similar diagnoses of atrioventricular node re-entry tachycardia (AVNRT) or a tachycardia substrate consisting of an accessory pathway or pathways. The first study group consisted of patients whose procedure involved the use of the EnSite system and fluoroscopy, and the comparison group's procedure consisted of fluoroscopy alone. In the fluoroscopy-alone group, the mean fluoroscopy time per procedure was $24.9 \mathrm{~min}$. In the fluoroscopy plus mapping group, the fluoroscopy time per procedure was cut by more than half, with a mean fluoroscopy time of $10.4 \mathrm{~min}$ $(p<0.0001)$. There were no differences in tachycardia substrates, success rates, or number of radiofrequency lesions delivered. Furthermore, the mean procedure time of the group using the system was significantly shorter (170 min versus $218 \mathrm{~min}, \mathrm{p}<0.0001$ ).

In a recent publication, Papagiannis and his colleagues $^{17}$ further reported outcomes using a minimal fluoroscopic technique in pediatric patients, based upon accessory pathway location, and in patients with abnormal cardiac structure. Again, this was a retrospective study consisting of a study group of patients whose procedure was performed with fluoroscopy and an electroanatomic mapping system compared with a group of patients whose procedure was carried out with fluoroscopy alone. The procedures in the minimal fluoroscopic approach group of patients had been carried out more recently. The procedures in the fluoroscopy-alone group were performed prior to the acquisition of the mapping system. Other than the period of time when the procedure was performed, the two groups were similar with respect to mean age, pathway location, and presence of congenital heart disease. There was a significant reduction in the mean fluoroscopy time in the group using the electroanatomic mapping system compared with the group with fluoroscopy alone, 8.27 versus $39.77 \mathrm{~min}(\mathrm{p}<0.001)$. Concomitant with the reduction in mean fluoroscopy time, the mean procedure duration was significantly shorter in the threedimensional mapping system group, $177.06 \mathrm{~min}$ versus $242.45 \mathrm{~min}(\mathrm{p}<0.001)$. The difference between the two groups was statistically significant in all accessory pathway locations. There were no complications attributable to the use of the electroanatomic mapping system. The acute success rates of the two groups were not different, nor was there a difference in the recurrence rate.

The issue of approaching the left atrium in pediatric patients without the use of fluoroscopy was addressed by Clark et al. ${ }^{18}$ In that report, 10 patients underwent cryoablation of left-sided pathways without fluoroscopy. A transseptal approach was used to gain access to the left atrium. As the transseptal sheath used does not have an electrode, it cannot be tracked by an electroanatomic mapping system. So, to enable a safe transseptal puncture, transesophageal echo was used to visualize the fossa ovalis. Tenting the fossa with the transseptal dilator was monitored. The transseptal needle was advanced into the left atrium and the location of the needle tip was confirmed on transesophageal echocardiogramy (TEE) by saline contrast injection. The sheath and dilator were advanced over the needle with continuous pressure monitoring and TEE. Once the sheath was appropriately positioned, the ablation was completed using only the electroanatomic mapping system for guidance. All patients had acutely successful ablations with no complications.

In the majority of studies noted in this review, often a comparison group comprised patients who had their procedure performed in an era prior to the acquisition of an electroanatomic mapping system. The cumulative 
experience of the operator(s) and laboratory personnel in performing electrophysiology studies and ablations could be a confounding factor in the measurement of the effects of the use of a non-fluoroscopic technique on procedural times and outcomes. Additionally, one would expect a learning curve to exist with the development of any new tool, and this has been noted with the development of electroanatomic mapping system-guided procedures. ${ }^{12}$

This effect of a learning curve was investigated in a laboratory where one experienced operator performed all pediatric ablations. ${ }^{19}$ Patients undergoing ablation of AVNRT were divided into an early and more recent era. The early era was defined as the first 12 months after the installation of a mapping system. The more recent era consisted of the ensuing 18 months. There were 27 patients in the early era and 35 patients in the recent era. No fluoroscopy was used in any procedures. Acute procedural success rates, long-term success rates, and complication rates were not different between the two eras. However, average procedure time for the early era was $202 \mathrm{~min}$ compared with $160 \mathrm{~min}$ in the more recent era $(p=0.01)$. The authors further noted that the shortening of the procedure by a mean of $42 \mathrm{~min}$ was not related to the time it took the laboratory staff to set the room for the procedure, but was related to the operators comfort with manipulating catheters within the body using tactile feedback and images provided by the electroanatomic mapping system.

Recently a descriptive study using CartoSound, an electroanatomic computer-assisted mapping system with the ability to integrate real-time intracardiac echocardiographic (ICE) images into the geometry, was published. ${ }^{20}$ The authors reviewed the first 17 patients whose procedures were performed using the new system in their laboratory. Their population comprised pediatric patients and patients with congenital heart disease. The majority of patients in this group had intra-atrial reentrant tachycardia (IART). The procedures on these patients were carried out without significant complication and with a high success rate. A subgroup consisting of the patients with IART was compared with a group of contemporary patients with IART carried out without CartoSound. Although procedure times, fluoroscopy times, and acute success rates were not significantly different, the authors concluded that there were advantages to using the mapping system. Specifically, they noted that they were able to create a more accurate geometry of the chamber of interest. Additionally, the system allowed the demonstration of catheter position and lesions on echo during the study. The authors felt that this allowed for the creation of better lines of block in complex anatomy.

Also, using the integration of ICE and electroanatomic mapping with the NavX system in their study group, Miyake and colleagues ${ }^{21}$ demonstrated a mean fluoroscopy time reduction by $59 \%$ over a group using fluoroscopy alone (18.3 $\mathrm{min}$ versus $7.5 \mathrm{~min}, \mathrm{P}<.001)$. Success rates and complication rates were not different between the two groups. Two patients in the study group had no fluoroscopy and two had less than $30 \mathrm{~s}$ of fluoroscopy. However, in the study group, mean procedure times were prolonged by $31 \mathrm{~min}$.

In addition to supraventricular tachycardia, computer electroanatomic mapping systems have been used to map and ablate ventricular dysrhythmias in pediatric patients with minimal or no fluoroscopy. ${ }^{22}$ In a report of five patients, three had right ventricular arrhythmias and the remaining two had left ventricular arrhythmias. The three patients with right ventricular foci were ablated with no fluoroscopy. Coronary angiography was required for patients with left ventricular foci and this accounted for the use of fluoroscopy. Catheter manipulation for mapping and ablation was carried out without fluoroscopy. This report also describes the technique the authors used for advancing long guiding sheaths without fluoroscopy. They took advantage of the change in the displayed catheter geometry based upon impedance changes when the electrodes were inside a sheath versus when they were out of the end of the sheath.

\section{Summary}

There is a growing body of literature that demonstrates safety and efficacy of minimal or no fluoroscopy use in pediatric patients undergoing ablation procedures by using an electroanatomic mapping system. In most studies, this has been accomplished without extending procedure times. However, the use of an electroanatomic mapping system increases the cost of a procedure over fluoroscopy alone. Despite that, concerns about longterm effects of radiation exposure on patients and operators are certainly part of the reason the use of these systems has grown. The extent to which long-term benefits outweigh the increased costs remains unknown.

In contrast to studies in adult patients, the majority of reports in the pediatric literature are single center, retrospective, and non-randomized. ${ }^{23,24}$ However, there is growing interest in studying the use of minimal or no fluoroscopy for ablation procedures on an organized, larger scale. At the Heart Rhythm Society's Scientific Sessions in 2010 a group of six investigators, many of whom were cited in this review, met informally. As a result of this group's efforts, the 2011 Heart Rhythm Society's Scientific Sessions had a formal session devoted to issues around non-fluoroscopic procedures. Again, an informal meeting open to all interested parties occurred after the formal session. The attendance at this informal meeting was fivefold larger than the initial meeting, involving adult and pediatric electrophysiologists. The result of that meeting is an effort at creating a multicenter database for minimal or non-fluoroscopic ablation procedures. At the time of this review, protocols for submission to this database are being prepared for individual institutional review board approval.

Although we are early in the formal investigation of the use of these electroanatomic mapping systems in pediatric patients, it is clear that interest in their use is growing rapidly. Technology development is also occurring at a rapid pace. It is foreseeable that the development of better 
techniques allowing shorter procedure times, savings in equipment purchase and maintenance, and decreased sequelae of ionizing radiation exposure will mitigate the current increased costs of using an electroanatomic mapping system.

\section{References}

1. Limacher MC, Douglas PS, Germano G, et al. ACC expert consensus document. Radiation safety in the practice of cardiology. American College of Cardiology. I Am Coll Cardiol 1988; 31:892-913.

2. U.S. Department of Health and Human Services. FDA unveils initiative to reduce unnecessary radiation exposure from medical imaging. 2010; https://wayback.archiveit.org/7993/20170112215923/http:/ / www.fda.gov/News Events/Newsroom/PressAnnouncements/2010/ucm 200085. htm.

3. Kim KP, Miller DL, Balter S, et al. Occupational radiation doses to operators performing cardiac catheterization procedures. Health Phys 2008; 94:211-227.

4. Wong L, Rehm J. Radiation injury from a fluoroscopic procedure. N Engl J Med; 350:e23.

5. Boncher J, Bergfeld WF. Fluoroscopy-induced chronic radiation dermatitis: a report of two additional cases and a brief review of the literature. J Cutan Pathol 2011; 1:1-5.

6. Ferguson JD, Helms A, Mangrum JM, et al. Catheter ablation of atrial fibrillation without fluoroscopy using intracardiac echocardiography and electroanatomic mapping. Circ Arrhythm Electrophysiol 2008; 2:611-619.

7. Ferguson JD, Helms A, Mangrum M, DiMarco JP. Ablation of Incessant Left Atrial Tachycardia without fluoroscopy in a pregnant woman. J Cardiovasc Electrophysiol 2011; 22:346-349.

8. Tuzcu V. A nonfluoroscopic approach for electrophysiology and catheter ablation procedures using a three-dimensional navigation system. Pacing Clin Electrophysiol 2007; 30:519-525.

9. Justino $H$. The ALARA concept in pediatric cardiac catheterization: techniques and tactics for managing radiation dose. Pediatr Radiol 2006; 36(Suppl)2:146-153.

10. Wagner LK. Minimizing radiation injury and neoplastic effects during pediatric fluoroscopy: what should we know? Pediatr Radiol 2006; 36(Suppl 2):141-145

11. Clay MA, Campbell RM, Strieper M, et al. Long-Term risk of fatal malignancy following pediatric radiofrequency ablation. Am J Cardiol 2008;102:913-915.

12. Drago F, Silvetti MS, Di Pino A, et al. Exclusion of fluoroscopy during ablation treatment of right accessory pathway in children. J Cardiovasc Electrophysiol 2002; 13:778782.

13. Kugler JD, Danforth DA, Houston KA, et al. Pediatric radiofrequency catheter ablation registry success, fluoro- scopy time, and complication rate for supraventricular tachycardia: comparison of early and recent eras. J Cardiovasc Electrophysiol 2002; 13:336-341.

14. Papez AL, al-Ahdab M, Dick M, Fischbach PS. Impact of a computer assisted navigation system on radiation exposure during pediatric ablation procedures. I Interv Card Electrophysiol 2007; 19:121-127.

15. Smith G, Clark JM. Elimination of fluoroscopy use in a pediatric electrophysiology laboratory utilizing threedimensional mapping. Pacing Clin Electrophysiol 2007; 30: 510-518.

16. Papagiannis J, Tsoutsinos A, Kirvassilis G, et al. Catheter Ablation of Supraventricular Tachycardia in Children. Pacing Clin Electrophysiol 2006; 29:971-978.

17. Papagiannis J, Vramidis DA, Alexopoulos C, Kirvassilis G. Radiofrequency ablation of accessory pathways in children and congenital heart disease patients: impact of a nonfluoroscopic navigation system. Pacing Clin Electrophysiol 2011; 1:1-9.

18. Clark J, Bockoven JR, Lane J, et al. Use of three-dimensional catheter guidance and trans-esophageal echocardiography to eliminate fluoroscopy in catheter ablation of $\mathrm{f}$ left-sided accessory pathways. Pacing Clin Electrophysiol 2008; 31:283289.

19. Gist K, Tigges C, Smith G, Clark J. Learning curve for zerofluoroscopy catheter ablation of AVNRT: early versus late experience. Pacing Clin Electrophysiol 2011; 34:264-268.

20. Kean AC, Gelehrter SK, Shetty I, et al. Experience with CartoSound for arrhythmia ablation in pediatric and congenital heart disease patients. J Interv Card Electrophysiol 2010; 29:139-145.

21. Miyake CY, Mah DY, Atallah J, et al. Nonfluoroscopic imaging systems reduce radiation exposure in children undergoing ablation of supraventricular tachycardia. Heart Rhythm 2011; 519-525. (http://www.sciencedirect.com/science/ article/pii/S154752711001386X-cor1\#cor1 http://www. sciencedirect.com/science/article/pii/S154752711001386Xfoot1\#foot1)

22. Von Bergen NH, Bansal S, Gingerich J, Law IH. Nonfluoroscopic and radiation-limited ablation of ventricular arrhythmias in children and young adults: A case series. Pediatr Cardiol 2011; 32:743-747.

23. Hindricks G, Piorkowski C, Risius T, et al. Effect of electroanatomically guided versus conventional catheter ablation of typical atrial flutter on the fluoroscopy time and resource use: A prospective randomized multicenter study. J Cardiovasc Electrophysiol 2009; 20:734-740.

24. Khaykin Y, Oosthuizen R, Zarnett L, et al. CARTO-guided vs. NavX-guided pulmonary vein antrum isolation and pulmonary vein antrum isolation performed without 3-D mapping: effect of the 3-D mapping system on procedure duration and fluoroscopy time. J Interv Card Electrophysiol $2011 ; 30: 233-240$. 